\title{
Analysis of the Status Quo and Influencing Factors of Readiness for Hospital Discharge of Patients Undergoing Hysteromyomectomy
}

\author{
Liu Zhang, Gaijing Wang, Ting Cai, Yanling Heng* \\ Department of Medicine, Yangtze University, Jingzhou, China \\ Email: ^575966059@qq.com
}

How to cite this paper: Zhang, L., Wang, G.J., Cai, T. and Heng, Y.L. (2020) Analysis of the Status Quo and Influencing Factors of Readiness for Hospital Discharge of Patients Undergoing Hysteromyomectomy. Yangtze Medicine, 4, 173-182. https://doi.org/10.4236/ym.2020.43017

Received: November 12, 2019

Accepted: June 27, 2020

Published: June 30, 2020

Copyright $\odot 2020$ by author(s) and Scientific Research Publishing Inc. This work is licensed under the Creative Commons Attribution International License (CC BY 4.0).

http://creativecommons.org/licenses/by/4.0/

\section{(c) (i) Open Access}

\begin{abstract}
Objective: To evaluate the status quo and influencing factors of readiness for hospital discharge of patients undergoing hysteromyomectomy. Methods: A total of 240 patients with uterine fibroid undergoing hysteromyomectomy from 2 hospitals in Jingzhou were investigated using a self-designed general information questionnaire, the Readiness for Hospital Discharge Scale and the Quality of Discharge Teaching Scale. Results: The total score of readiness for hospital discharge was $(91.36 \pm 18.46)$, the multiple linear regression analysis showed that the quality of discharge guidance, the scope of myomectomy, pain degree of incision and the average monthly income per family were the main influencing factors of readiness for hospital discharge. Conclusion: The readiness for hospital discharge was at a medium level in patients with uterine fibroid undergoing hysteromyomectomy, medical personnel should give specific discharge guidance according to the specific conditions of patients to ensure the safety of patients after discharge.
\end{abstract}

\section{Keywords}

Uterine Fibroid, Readiness for Discharge, Discharge Teaching, Influencing Factors

\section{Introduction}

The readiness for hospital discharge was first proposed by Fenwick [1] in 1976, it refers to the ability of patients to cope in the community after transitioning from an acute care hospital, it's a kind of self-perception of whether patients are ready for discharge. As a mediation variable in the transition period from discharge to return home, the readiness for hospital discharge is closely related to the effect of rehabilitation, quality of life and outcome after discharge [2]. Surgical treatment 
is the main treatment for symptomatic uterine fibroid, but the current treatment is more or less accompanied by trauma and postoperative complications. Some studies have shown that the risk of postoperative recurrence of uterine fibroid is $4 \%$ - 30\%, among which $10 \%$ patients still need further intervention after hysteromyomectomy, some patients performed high stress response due to surgery: anxiety, depression, fear, etc., so there will still be different degrees of health problems after discharge from hospital and there is a high demand for health care [3] [4] [5] [6]. The readiness of hospital discharge is related to patients' self-care ability, and has a significant impact on improving patients' self-efficacy and quality of life after discharge. To investigate the level of readiness for hospital discharge level of patients after hysteromyoma operation, 240 patients were investigated, and the influencing factors were analyzed, so as to provide scientific basis for improving the self-care ability of patients and developing standardized discharge guidance in clinical.

\section{Materials and Methods}

\subsection{Patients}

240 patients with uterine fibroid undergoing hysteromyomectomy from December 2018 to June 2019 in the work were included. Inclusion criteria: it conforms to the diagnostic standards of uterine fibroid by "Chinese Journal of obstetrics and gynecology" [7], accepted the surgical treatment; hospital stay $\geq 3$ days; discharge with medical advice; voluntarily participated in the study. Exclusion criteria: with communication barriers, unclear consciousness or mental disorder; with other important organ function damage. This study has been approved by the head nurses of the nursing departments.

\subsection{Research Tools}

1) A general information questionnaire was designed by the researchers according to the research purpose, including 15 basic information such as general demographic data (age, residence, education level, marital status, working status and mode of payment), disease-related data (reason for medical consultations, operation method, scope of myomectomy and so on).

2) The Readiness for hospital discharge scale (RHDS) was developed by Marianne et al. [8] in 2006. In this study, the Chinese version of RHDS translated and revised by Lin Youhua et al. [1], a Taiwan scholar of China, was used. After translation and reliability and validity test, the scale only contains 12 items based on the original scale. The items from 3 sub-scales: personal status, coping ability and expected support, scored on a 10-point scale $(0-10)$. Higher scores indicate greater readiness. The Cronbach's $\alpha$ coefficient of the Chinese scale is 0.89 , the content validity index is 0.88 , and the content validity index of each item is $0.80-1$, which is highly correlated with the original scale.

3) The quality of discharge teaching scale (QDTS) was developed by Weiss et al. [2]. In this study, the Chinese version of QDTS translated and revised by 
Wang Binghua et al. [9] was used. The scale retained all the items and structures of the original scale, including 24 items, 3 sub-scales: the amount of "content needed", "content received" and "teaching skills". The total scale score is calculated by adding the content received and the delivery sub-scale scores. The content validity index was 0.98 , the Cronbach's $\alpha$ coefficients of the total scale and 3 factors were 0.924 , and 0.882 - 0.935 . The Chinese version of QDTS is reliable and valid and can be used for assessment and measurement of the quality of discharge teaching in Chinese settings.

\subsection{Data Collection}

In this study, relevant data were collected by sending questionnaires to discharged patients who met the inclusion criteria. The questionnaire survey was carried out on the day before the patient discharged from the hospital. 260 questionnaires were issued and 240 effective questionnaires were recovered, the effective recovery rate was $92.31 \%$.

\subsection{Statistical Analysis}

SPSS22.0 software was used for statistical analysis. The general data of patients are expressed by frequency and percentage, and the scores of each scale are analyzed by Mean \pm SD. The results of single factor analysis are compared by $t$-test or variance analysis, Pearson correlation analysis is carried out between the two scales. 95\% CI was calculated for all analyses $(\alpha=0.05)$.

\section{Results}

\subsection{Comparison of Readiness of Hospital Discharge Scores of Patients with Different Demographic Characteristics}

The results of single factor analysis show that in demographic data, the education level and monthly income of family are the influencing factors of readiness of hospital discharge $(P<0.05)$. The difference of readiness of hospital discharge scores of patients with different reasons for medical consultations, scope of myomectomy, operative method, pain degree of incision and day after operation was statistically significant $(P<0.05)$ (Table 1$)$.

\subsection{The Status Quo of Readiness for Hospital Discharge}

The mean total score of RHDS in this study was (91.36 \pm 18.46$)$, in a Medium level. The standardized scores in the three subscales were: personal status $(7.48 \pm$ 1.87), coping ability (7.67 \pm 1.84$)$, and expected support $(7.70 \pm 2.04)$ (Table 2).

\subsection{The Status of the Quality of Discharge Teaching}

In the study, the mean total score of QDTs was (136.94 \pm 26.50$)$, while the standardized scores were higher for the received subscale $(6.23 \pm 2.26)$ than for the Content needed subscale $(5.75 \pm 2.55)$, indicated that the patient's care needs were met (Table 3 ). 
Table 1. Comparison of readiness of hospital discharge scores of patients $(n=240)$.

\begin{tabular}{|c|c|c|c|c|c|}
\hline Characteristic & Grade & $\begin{array}{c}\text { Number } \\
(\%)\end{array}$ & $\begin{array}{l}\text { Readiness } \\
\left(\begin{array}{ll}\bar{X} & \pm S\end{array}\right)\end{array}$ & $\begin{array}{l}\text { Statistic } \\
\text { value }\end{array}$ & $P$-value \\
\hline \multirow[t]{3}{*}{ Residence } & Urban & $61(25.42)$ & $92.67 \pm 17.79$ & $0.407^{1)}$ & 0.666 \\
\hline & Town & $68(28.33)$ & $92.04 \pm 17.02$ & & \\
\hline & Countryside & $111(46.25)$ & $90.23 \pm 19.80$ & & \\
\hline \multirow[t]{4}{*}{ Age (years) } & $\leq 35$ & $62(25.83)$ & $95.98 \pm 16.82$ & $2.427^{1)}$ & 0.066 \\
\hline & $36-45$ & $81(33.75)$ & $89.78 \pm 20.77$ & & \\
\hline & $46-55$ & $74(30.83)$ & $91.19 \pm 17.21$ & & \\
\hline & $\geq 56$ & $23(9.58)$ & $85.04 \pm 16.52$ & & \\
\hline \multirow[t]{5}{*}{ Education } & Primary or below & $38(15.83)$ & $85.69 \pm 18.32$ & $2.683^{1)}$ & 0.032 \\
\hline & Junior middle school & $108(45.00)$ & $89.82 \pm 19.79$ & & \\
\hline & Secondary/High school & $34(14.17)$ & $93.24 \pm 19.03$ & & \\
\hline & College & $37(15.42)$ & $95.54 \pm 16.26$ & & \\
\hline & Bachelor degree or above & $22(9.17)$ & $99.05 \pm 10.00$ & & \\
\hline \multirow[t]{3}{*}{ Marital status } & Single & $12(5.00)$ & $91.58 \pm 16.96$ & $0.365^{1)}$ & 0.694 \\
\hline & Married & $215(89.58)$ & $91.09 \pm 18.74$ & & \\
\hline & Divorce/Widowhood & $13(5.42)$ & $95.62 \pm 16.46$ & & \\
\hline \multirow[t]{3}{*}{ Working state } & Incumbency & $118(49.17)$ & $94.03 \pm 17.85$ & $2.267^{1)}$ & 0.081 \\
\hline & Retired & $23(9.58)$ & $87.35 \pm 11.91$ & & \\
\hline & Unemployed & $98(40.83)$ & $88.87 \pm 20.08$ & & \\
\hline \multirow{5}{*}{$\begin{array}{l}\text { Mode of } \\
\text { payment }\end{array}$} & All voluntary spends & $16(6.67)$ & $96.56 \pm 15.66$ & $1.564^{1)}$ & 0.185 \\
\hline & Public expense & $2(0.83)$ & $100.50 \pm 3.54$ & & \\
\hline & $\begin{array}{l}\text { New rural cooperative } \\
\text { medical insurance }\end{array}$ & $119(49.58)$ & $89.19 \pm 19.30$ & & \\
\hline & Other medical insurance & $95(38.58)$ & $93.65 \pm 16.41$ & & \\
\hline & Poverty seeking help & $8(3.33)$ & $83.75 \pm 31.07$ & & \\
\hline \multirow{2}{*}{$\begin{array}{l}\text { Reasons for } \\
\text { medical }\end{array}$} & Physical examination & $117(48.75)$ & $93.97 \pm 17.03$ & $4.850^{2)}$ & 0.029 \\
\hline & Clinical feature & $123(51.25)$ & $88.98 \pm 19.63$ & & \\
\hline \multirow{4}{*}{$\begin{array}{c}\text { Scope of } \\
\text { myomectomy }\end{array}$} & Total hysterectomy & $60(25.00)$ & $84.37 \pm 18.73$ & $5.374^{1)}$ & 0.001 \\
\hline & Partial hysterectomy & $153(63.75)$ & $93.54 \pm 17.74$ & & \\
\hline & Myomectomy & $21(8.75)$ & $90.86 \pm 19.57$ & & \\
\hline & Others & $6(2.50)$ & $107.50 \pm 8.87$ & & \\
\hline \multirow{4}{*}{$\begin{array}{l}\text { Operative } \\
\text { method }\end{array}$} & Laparotomy & $64(26.67)$ & $84.69 \pm 18.37$ & $5.182^{1)}$ & 0.002 \\
\hline & Laparoscope & $24(10.00)$ & $93.21 \pm 19.27$ & & \\
\hline & Hysteroscopy & $146(60.83)$ & $93.32 \pm 17.92$ & & \\
\hline & Ultrasonic focusing & $6(2.50)$ & $107.50 \pm 8.87$ & & \\
\hline \multirow{2}{*}{$\begin{array}{l}\text { Pain degree } \\
\text { of incision }\end{array}$} & 0 & $61(25.42)$ & $98.59 \pm 14.49$ & $7.368^{1)}$ & $<0.001$ \\
\hline & 1 & $75(31.25)$ & $94.92 \pm 16.98$ & & \\
\hline
\end{tabular}




\section{Continued}

\begin{tabular}{|c|c|c|c|c|c|}
\hline & 2 & 77 (32.08) & $87.17 \pm 19.28$ & & \\
\hline & 3 & $20(8.33)$ & $78.35 \pm 20.07$ & & \\
\hline & 4 & $6(2.50)$ & $74.50 \pm 13.10$ & & \\
\hline & 5 & $1(0.42)$ & 68.00 & & \\
\hline Day after & $\leq 3$ & $36(15.00)$ & $90.69 \pm 20.53$ & $4.736^{1)}$ & 0.010 \\
\hline operation & $4-7$ & $161(67.08)$ & $93.50 \pm 17.40$ & & \\
\hline & $\geq 8$ & 43 (17.92) & $83.91 \pm 19.21$ & & \\
\hline Admission & 1 & $197(82.08)$ & $92.03 \pm 18.34$ & $2.754^{1)}$ & 0.066 \\
\hline times & 2 & $34(14.17)$ & $91.24 \pm 19.74$ & & \\
\hline & $>2$ & $9(3.75)$ & $77.33 \pm 11.97$ & & \\
\hline Chronic & No & $209(87.08)$ & $92.24 \pm 18.16$ & $2.360^{1)}$ & 0.097 \\
\hline disease & 1 & $24(10.00)$ & $87.21 \pm 22.13$ & & \\
\hline & $\geq 2$ & $7(2.92)$ & $79.29 \pm 7.39$ & & \\
\hline Monthly & $\leq 2000$ & $50(20.83)$ & $88.56 \pm 19.58$ & $6.512^{1)}$ & $<0.001$ \\
\hline income (RMB) & $2000-4000$ & $78(32.50)$ & $85.14 \pm 19.47$ & & \\
\hline & $4000-6000$ & $67(27.92)$ & $94.72 \pm 17.34$ & & \\
\hline & $6000-9000$ & $28(11.67)$ & $97.86 \pm 11.30$ & & \\
\hline & $\geq 9000$ & $17(7.08)$ & $104.24 \pm 12.18$ & & \\
\hline Primary & Children/Husband & $186(77.50)$ & $91.33 \pm 18.75$ & $2.012^{1)}$ & 0.136 \\
\hline caregiver & Self & $32(13.33)$ & $87.31 \pm 18.88$ & & \\
\hline & Others & $22(9.17)$ & $97.55 \pm 14.40$ & & \\
\hline
\end{tabular}

${ }^{1)} F$-value, ${ }^{2)} t$-value.

Table 2. Readiness for hospital discharge of patients after hysteromyoma ( $\mathrm{n}=240, \bar{X} \pm$ S).

\begin{tabular}{ccccc}
\hline Sub-scales & Number of items & Full score & Actual score & Standardized score \\
\hline Personal status & 4 & 40 & $29.92 \pm 7.46$ & $7.48 \pm 1.87$ \\
Coping ability & 4 & 40 & $30.67 \pm 7.35$ & $7.67 \pm 1.84$ \\
Expected support & 4 & 40 & $30.78 \pm 8.14$ & $7.70 \pm 2.04$ \\
Total score & 12 & 120 & $91.36 \pm 18.46$ & $7.61 \pm 1.54$ \\
\hline
\end{tabular}

Table 3. The quality of discharge teaching of patients after hysteromyoma ( $\mathrm{n}=240, \bar{X}$ \pm S).

\begin{tabular}{ccccc}
\hline Sub-scales & Number of items & Full score & Actual score & Standardized score \\
\hline Content needed & 6 & 60 & $34.48 \pm 15.28$ & $5.75 \pm 2.55$ \\
Content received & 6 & 60 & $37.38 \pm 13.57$ & $6.23 \pm 2.26$ \\
Guidance skills and effect & 12 & 120 & $99.56 \pm 18.25$ & $8.30 \pm 1.52$ \\
Total score & 18 & 180 & $136.94 \pm 26.50$ & $7.61 \pm 1.47$ \\
\hline
\end{tabular}




\subsection{Correlation Analysis of Readiness for Hospital Discharge and the Quality of Discharge Teaching of Patients}

The scores of QDTS and its correlation with RHDS: in the study, there were statistically significant relativity between RHDS score and both total scale score and most subscales scores of QDTS $(P<0.05)$ (Table 4$)$.

\subsection{Analysis of the Influencing Factors of Readiness for Hospital Discharge of Patients}

Taking the total score of readiness for hospital discharge of patients as the dependent variable, 7 variables with statistical significance in single factor analysis were used as independent variables for multiple linear regression analysis. Variable assignments were as follows: 1) Education: Primary or below $=1$, Junior middle school $=2$, Secondary specialized or high school $=3$, College $=4$, University $=5$;2) Reasons for medical consultations: physical examination $=1$, clinical features $=2$; 3) Scope of myomectomy: Total hysterectomy $=1$, Partial hysterectomy $=2$, Myomectomy $=3$, Others $=4 ; 4$ ) Operative method: Laparotomy $=1$, Laparoscope $=2$, Hysteroscopy $=3$, Ultrasonic focusing $=4 ; 5$ ) Pain degree of incision: $0=1,1=2,2=3,3=4,4=5 ; 6)$ Day after operation: $\leq 3$ days $=1,4$ 7 days $=2, \geq 8$ days $=3$; 7) Monthly income of family: $\leq 2000=1,2000-4000=$ $2,4000-6000=3,6000-9000=4, \geq 9000=5$. The results showed that monthly income of family, pain degree of incision, uterine fibroid resection and the quality of discharge teaching were the main influencing factors of readiness for hospital discharge (Table 5).

Table 4. Correlation analysis of two scales $(\mathrm{n}=240, r$-value).

\begin{tabular}{ccccc}
\hline & $\begin{array}{c}\text { Total score } \\
\text { of discharge } \\
\text { readiness }\end{array}$ & $\begin{array}{c}\text { Personal } \\
\text { status }\end{array}$ & $\begin{array}{c}\text { Coping } \\
\text { ability }\end{array}$ & $\begin{array}{c}\text { Expected } \\
\text { support }\end{array}$ \\
Content needed & -0.113 & -0.140 & $-0.168^{*}$ & 0.015 \\
Content received & $0.387^{*}$ & $0.273^{*}$ & $0.348^{*}$ & $0.323^{*}$ \\
Guidance skills and effect & $0.464^{*}$ & $0.390^{*}$ & $0.432^{*}$ & $0.322^{*}$ \\
Total score of discharge guidance quality & $0.491^{*}$ & $0.397^{*}$ & $0.450^{*}$ & $0.361^{*}$ \\
\hline
\end{tabular}

${ }^{\star} P<0.05$.

Table 5. Multiple linear regression analysis of readiness for hospital discharge $(n=240)$.

\begin{tabular}{ccccc}
\hline & $\begin{array}{c}\text { Regression } \\
\text { coefficient }\end{array}$ & $\begin{array}{c}\text { Standardized } \\
\text { regression } \\
\text { coefficient }\end{array}$ & $t$-value & $P$-value \\
\hline Constant term & 41.278 & - & 4.745 & 0.000 \\
Scope of myomectomy & 3.901 & 0.189 & 2.729 & 0.007 \\
Pain degree of incision & -3.701 & -0.202 & -2.759 & 0.006 \\
Monthly income of family & 3.134 & 0.159 & 2.394 & 0.018 \\
Total of the quality of discharge teaching & 0.280 & 0.412 & 5.880 & $<0.001$ \\
\hline
\end{tabular}

$F=5.730, P<0.05, R^{2}=0.363$, adjusted $R^{2}=0.345$. 


\section{Discussion}

\subsection{The Readiness for Hospital Discharge of Patients with Hysteromyoma Is at a Medium Level}

Item 1 of the readiness for hospital discharge scale is true or false, $93.75 \%$ (225 cases) of patients think they are prepared for discharge. However, only $68.75 \%$ of the patients have an average score of more than 7, according to Weiss et al. [10], the standardized score of readiness for hospital discharge is less than 7 , which is a low-level threshold, probably because the patient's worry about hospital expenses. Even if they are not ready for discharge, they still answer that they are prepared, or some patients have cognitive bias and overestimate their preparation for discharge [11]. In this study, the total score of the readiness for hospital discharge of patients with hysteromyoma was (91.36 \pm 18.46$)$, and the average score of items was $(7.61 \pm 1.54)$, in a middle level. The results were similar to those of patients after radical operation of cervical cancer [12] (7.70 \pm 1.03$)$ and hepatobiliary surgery [13] $(7.28 \pm 1.64)$. The scores of 3 dimensions from high to low were expected support, coping ability and personal status, which may be related to the following factors: 1) The overall education level of the patients included in this study was low (60.83\% were junior middle school or below), and about $46 \%$ of the patients lived in rural areas, so many of them lack the knowledge of disease rehabilitation, compared with cities and towns, rural areas have a narrow access to information platform and less social support. Study [14] shown that $70.9 \%$ of women have poor overall understanding of hysteromyoma and its treatment, with an average score of less than $60 \%$. 2) The recovery of hysteromyoma after operation is a long-term process, some patients are damaged in physical integrity, and they have insufficient understanding or psychological preparation on how to deal with the disease recovery after discharge. It is suggested that medical staff should pay attention to the difference of patients' needs in health education for patients with hysteromyoma after operation, especially the patients with low education level and living in rural areas, and implement individualized health education programs to meet their needs.

\subsection{The Readiness for Hospital Discharge of Patients with Hysteromyoma Is Affected by Various Factors}

1) The readiness for hospital discharge of patients with hysteromyoma after operation is affected by the reasons for medical consultations, and monthly income of the family.

The results of this study show that the score of the readiness for hospital discharge of patients who seek for medical treatment for physical examination is higher than those who seek medical treatment due to clinical symptoms. The reason may be that the patients who find medical treatment due to physical examination have no or less clinical manifestations, and have higher awareness of their own health care. The monthly income of the family is significantly related to their personal status and coping ability $(P<0.001)$, the higher the monthly 
income of the patients, the higher the level of the discharge preparation. The reasons may be that the patients with good economic conditions can actively seek medical help, have better medical security method and less economic burden, can focus on postoperative rehabilitation after discharge quickly. Higher monthly income is a protect factor for discharge readiness, which can better meet the health needs of the patients [15].

2) The readiness for hospital discharge of patients with hysteromyoma after operation is affected by scope of myomectomy, operative method and pain degree of incision.

The results of this study show that the score of readiness for hospital discharge of patients with hysteromyoma treated by high-intensity focused ultrasound therapy is higher, and that of patients treated with hysteromyoma or laparoscopy is the second, and that of patients with open total hysterectomy is the lowest. On the day of discharge, the patients with lower incision pain degree have higher discharge readiness level. As an extracorporeal therapy, high intensity focused ultrasound therapy is simple and has no damage or wound to normal tissues, and patients can quick recovery postoperatively [16]. Patients undergoing laparotomy recovery slowly, their self-care ability is reduced, so most of them have lower readiness for hospital discharge level. Operative method and pain degree of incision were also the main influencing factors of patients' personal status and coping ability $(P<0.05)$. Giving nursing intervention to patients can relieve the pain of incision and keep patients in a best state of mind and body, and promote the recovery [17].

3) The readiness for hospital discharge of patients with hysteromyoma after operation is closely related to the quality of discharge teaching.

The results of this study showed that the quality of discharge teaching for patients with hysteromyoma after operation was at a medium level (136.94 \pm 26.50) and positively correlated with the readiness for hospital discharge ( $r=$ $0.491, P<0.05)$. The score of the guidance skills and effect is highest, it may be that the investigation department of the institutes teach the health knowledge to patients and their families in one day of a week. In the other two dimensions, the score of the content needed and actual received were relatively low. The reason for the analysis may be that the discharge teaching scale is evaluated by the patient subjectively, which need them to fill in by connecting the expectation with practice, however some patients have cognitive bias and so it is easy to make a wrong judgment if they do not understand their own situation. In addition, this study also found that operative method and whether there are other chronic diseases have a significant impact on the content of patients' needs (all $P<0.05$ ). Patients who undergoing laparotomy or total hysterectomy or have other chronic diseases have a higher demand for related health knowledge. Compared with patients themselves, medical staffs have a more comprehensive understanding and objective evaluation about patients, suggesting that medical staff should give targeted discharge guidance, not only to meet the functional needs of patients, but also to pay attention to their acceptance and utilization of informa- 
tion. The complete readiness for hospital discharge includes physical stability, sufficient support, psychological ability and enough information and knowledge. It suggests that medical staff should strengthen communication with patients and their families, provide them with high-quality discharge guidance services, and ensure that patients understand and master relevant contents of discharge guidance [18].

\subsection{Limitations}

1) If regarded this study as a descriptive cross-sectional study, the sample size is small and limited to a certain city, which cannot replace the situation of large areas, resulting in a decline in the persuasiveness of the results. 2) The questionnaire is collected in the hospital, and most of it is issued and recovered by the patient's responsible nurses, so there may be some inaccuracies of the data, which may bias the results.

\section{Conclusion}

The readiness for hospital discharge of patients with hysteromyoma after operation is at a medium level, which needs to be improved. Monthly income of family, scope of myomectomy, pain degree of incision and the quality of discharge teaching quality are the main influencing factors. For patients with poor economic status, received laparotomy or hysterectomy, we should pay more attention to them and strengthen the guidance. We can learn from Taiwan and foreign hospitals to try to carry out discharge preparation services to help patients prepare for discharge. At the same time, family members should be encouraged to give support to patients, mobilize their subjective initiative, and ensure the safety of patients after discharge. In the future study, we can understand the common problems through qualitative interviews that hinder patients' perception of discharge readiness, and formulate corresponding nursing interventions based on the problems. Through interviews with patients, combined with relevant literature and guidelines, we can develop standard discharge guidance process and content.

\section{Conflicts of Interest}

The authors declare no conflicts of interest regarding the publication of this paper.

\section{References}

[1] Lau, D., Padwal, R.S., Majumdar, S.R., et al. (2016) Patient-Reported Discharge Readiness and 30-Day Risk of Readmission or Death: A Prospective Cohort Study. The American Journal of Medicine, 129, 89-95. https://doi.org/10.1016/j.amjmed.2015.08.018

[2] Weiss, M.E., Piacentine, L.B., Lokken, L., et al. (2007) Perceived Readiness for Hospital Discharge in Adult Medical-Surgical Patients. Clinical Nurse Specialist, 21, 31-42. https://doi.org/10.1097/00002800-200701000-00008 
[3] Saridogan, E. (2016) Surgical Treatment of Fibroids in Heavy Menstrual Bleeding. Women's Health, 12, 53-62. https://doi.org/10.2217/whe.15.89

[4] Guo, J.L. (2012) Application and Effect Evaluation of Special Nursing Path in Patients Undergoing Hysteromyoma Operation. Chinese Journal of Practical Nursing, 28, 50-51.

[5] Ekine, A.A., Lawani, L.O., Iyoke, C.A., Jeremiah, I. and Ibrahim, I.A. (2015) Review of the Clinical Presentation of Uterine Fibroid and the Effect of Therapeutic Intervention on Fertility. American Journal of Clinical Medicine Research, 3, 9-13.

[6] Pan, Y.P. (2018) Effect of Evidence-Based Nursing on the Psychological State and Complications of Patients Undergoing Hysteromyomectomy. Today Nurse, 25, 97-98.

[7] Cao, Z.Y. (2010) Chinese Journal of Obstetrics and Gynecology. People's Medical Publishing House, Beijing, 1802-1829.

[8] Marianne, E., Weiss, M.E. and Linda, B. (2006) Psychometric Properties of the Readiness for Hospital Discharge Scale. Journal of Nursing Measurement, 14, 163-178. https://doi.org/10.1891/jnm-v14i3a002

[9] Wang, B.H., Wang, H., Wang, C., et al. (2016) Reliability and Validity of the Chinese Version of the Quality of Discharge Teaching Scale. Chinese Journal of Nursing, 51, 753-756.

[10] Weiss, M., Yakusheva, O. and Bobay, K. (2010) Nurse and Patient Perceptions of Discharge Readiness in Relation to Postdischarge Utilization. Medical Care, 48, 482-486. https://doi.org/10.1097/MLR.0b013e3181d5feae

[11] Liu, J.X., Wu, L.J., Song, Y.Q., et al. (2018) Postoperative Discharge Readiness and Influencing Factors of Breast Cancer Patients after Total Resection. Chinese Journal of Nursing Education, 15, 856-870.

[12] Gao, J., Gao, B.H., Wang, H., et al. (2019) Perceived Readiness for Hospital Discharge and Its Associated Factors in Cervical Cancer Patients Undergoing Radical Hysterectomy and Requiring Clean Intermittent Self-Catheterization. Journal of Nursing Science, 34, 16-18.

[13] Li, J., Tian, B.J., Yu, S., et al. (2019) Correlation between Readiness for Hospital Discharge and Quality of Discharge Teaching in Patients after Hepatobiliary Surgery. Journal of Nursing Science, 34, 85-87.

[14] Howard, D.L., Gopal, N., Stockwell, E. and Volker, K.W. (2018) Women's Health Literacy Regarding the Hysterectomy Procedure and Uterine Fibroids. American Journal of Obstetrics and Gynecology, 218, S909. https://doi.org/10.1016/j.ajog.2017.12.043

[15] Cai, Y.H., Yang, H., Wu, T., et al. (2019) Nursing Outcomes of Discharged Patients with Chronic Obstructive Pulmonary Disease and the Associate Factors. Journal of Nursing Science, 34, 85-87.

[16] Haipaiti, Z. (2014) Clinical Analysis on 130 Cases with Uterine Fibroid Adenomyosis Treated by High Intensity Focused Ultrasound. Xinjiang Medical University, Ürümqi, China.

[17] Cui, X.Q., Ding, P., Shi, X., et al. (2019) Effect of Nursing Intervention on Postoperative Pain and Quality of Life of Patients with Hysteromyoma. Electronic Journal of Practical Clinical Nursing Science, 4, 38-40.

[18] Galvin, E.C., Wills, T. and Coffey, A. (2017) Readiness for Hospital Discharge: A Concept Analysis. Journal of Advanced Nursing, 73, 2547-2557. https://doi.org/10.1111/jan.13324 